\title{
A Study of Surface Modifications of Carbon Nanotubes on the Properties of Polyamide 66/Multiwalled Carbon Nanotube Composites
}

\author{
Li Qiu, ${ }^{1,2}$ Yongkang Chen,,3 Yongzhen Yang, ${ }^{1,4}$ Lihua Xu, ${ }^{1,2}$ and Xuguang Liu ${ }^{1,2}$ \\ ${ }^{1}$ Key Laboratory of Interface Science and Engineering in Advanced Materials, Taiyuan University of Technology, \\ Ministry of Education, Taiyuan 030024, China \\ ${ }^{2}$ College of Chemistry and Chemical Engineering, Taiyuan University of Technology, Taiyuan 030024, China \\ ${ }^{3}$ University of Hertfordshire, School of Engineering and Technology, Hatfield, Hertfordshire AL10 9AB, UK \\ ${ }^{4}$ Research Center on Advanced Material Science and Technology, Taiyuan University of Technology, Taiyuan 030024, China
}

Correspondence should be addressed to Xuguang Liu; liuxuguang@tyut.edu.cn

Received 15 July 2013; Accepted 9 September 2013

Academic Editor: Raghavendra Hegde

Copyright ( $) 2013$ Li Qiu et al. This is an open access article distributed under the Creative Commons Attribution License, which permits unrestricted use, distribution, and reproduction in any medium, provided the original work is properly cited.

The effects of surface modification of carbon nanotube on the properties of polyamide 66/multiwalled carbon nanotube composites have been investigated. Polyamide 66 (PA66) and multiwalled carbon nanotube (MWCNT) composites were prepared by melt mixing. The surfaces of MWCNTs were modified with acid- and amine-groups. Field emission scanning electron microscopy analyses revealed that amine-MWCNTs (D-MWCNTs) dispersed better in the PA66 matrix than pristine- and acid-MWCNTs. However, an introduction of D-MWCNTs into PA66 matrix induced heterogeneous nucleation and affected the crystal growth process during the crystallization of PA66/MWCNT composites. Both nanoindentation and friction analyses were carried out in a study of the effect of the introduction of modified MWCNTs on both mechanical and friction properties of the composites. With the introduction of D-MWCNTs, both nanohardness and elastic modulus of the composites were significantly improved, but it was observed that the maximum depth, nanohardness, and elastic modulus of the composites showed no distinct change before and after a friction test. It is evident that PA66/D-MWCNT composites have the least friction coefficient of the PA66/MWCNT composites of all the approaches of carbon nanotube surface modification.

\section{Introduction}

For the past two decades, carbon nanotubes (CNTs) have attracted a great deal of attention in both academic and industrial aspects because of their remarkable self-lubricating, mechanical, electrical, and thermal properties, which allow them to be applied as high performance structure materials for aerospace and many other industrial fields [1-6]. Thus, CNTs are taken as good candidates for reinforcing a variety of polymers, ceramics, and metals. Among these promising materials, polymer/CNTs composites have been considered as one of the most attractive applications of CNTs with excellent performance and multifunction [7-11]. Moreover, the addition of CNTs can improve the mechanical and friction behavior of the polymer matrix. Recent investigations on the mechanics and tribology of CNTs filled polymer by Chiu and Kao [9] and Meng et al. [11] show a high hardness and elastic modulus and low friction. However, the challenges for improving performance of polymer/CNTs composites include improving dispersity of CNTs in polymer matrix and enhancing the interfacial interactions between polymeric matrix and CNTs to promote the load transfer from polymer matrix to CNTs. As a result of the small diameter and the chemically smooth surface of CNTs, they usually exhibit a highly aggregated state in common solvents and polymer matrix. Also, CNTs usually form stabilized bundles because of the van der Waals and electrostatic interactions between CNTs and matrix. Therefore, a homogeneous dispersion of CNTs in polymeric matrix is the key to obtain excellent performance of polymer/CNT composites. It is necessary to 
modify surface inertness of CNTs by functional groups or polymers that are either identical or structurally similar to matrix materials.

PA66 is a typical semicrystalline polymer and has been widely used in various engineering fields. PA66 is also the most important commercialized engineering plastic and possesses excellent chemical and abrasion resistance, plus low coefficient of friction, dimensional stability, toughness, and high hardness and strength. However, its lack of high temperature aging resistance and absorption of moisture in air lead to the inferior dimensional stability, which limits the applications of PA66. Both physical and mechanical properties of crystalline polymers are greatly dependent on the morphology, crystalline structure, and degree of crystallinity. Therefore modification and reinforcement of PA66 have drawn much attention to improve its physical properties and to introduce new properties. For example, Rodriguez-Pastor et al. [12] reported the preparation of helical-ribbon carbon nanofiber-based nylon-6,6 composites by three processing methods and the effect of CNTs modification methods on the properties of composites. Krause et al. [13] investigated the influence of CNTs production method and aspect ratio on percolation threshold of melt-mixed PA66.

This paper presents the new study outcomes in preparation of PA66/multiwalled carbon nanotube (MWCNT) composites by melt compounding using the twin-screw extruder; in microstructure characterization of different surfacemodified MWCNTs within the polymer matrix by field emission scanning electron microscopy (FESEM) and Fourier transform infrared spectrometry (FTIR); and in a systematical investigation of the effects of surface modification of carbon nanotube on both mechanical and friction properties of the obtained composites using different experimental tools.

\section{Experimental}

2.1. Preparation of PA66/MWCNT Composite. Pristine MWCNTs (out diameter: 30-50 nm; length: 10-20 $\mu \mathrm{m}$; purity $>95 \%$, P-MWCNTs) were treated with a mixture of $\mathrm{H}_{2} \mathrm{SO}_{4} /$ $\mathrm{HNO}_{3}(3: 1, \mathrm{v} / \mathrm{v})$ in an ultrasonic bath for 2 hours at $50^{\circ} \mathrm{C}$. MWCNTs were filtered and washed with deionized water until $\mathrm{pH}$ became 7 and dried in vacuum to give acid-treated MWCNTs (A-MWCNTs). A-MWCNTs were reacted with excessive $\mathrm{SOCl}_{2}$ under stirring at $70^{\circ} \mathrm{C}$ for 24 hours. The residual $\mathrm{SOCl}_{2}$ was then removed by filtration and washed with tetrahydrofuran. Subsequently, the solid product was dried under vacuum at $80^{\circ} \mathrm{C}$ for 12 hours to give acylchlorideMWCNTs (MWCNTs-COCl). Ethylenediamine (360 mL) was added to a three-neck flask that contained MWCNTs$\mathrm{COCl}(1.5 \mathrm{~g})$, and the mixture was stirred at $120^{\circ} \mathrm{C}$ for 48 hours. After the reaction, the product was vacuum-filtered and washed for several times with anhydrous ethanol and dichloromethane; thus the amine-modified MWCNTs (DMWCNTs) were obtained.

PA66/MWCNT composites were prepared by meltblending PA66 (101L) with MWCNTs. The melt compounding was performed in a melt-blending process using a $\mathrm{SHJ}-$ 36 twin-screw extruder. The temperature of the extruder was maintained at $250,260,260$, and $255^{\circ} \mathrm{C}$ from hopper to die, respectively. The rotation speed of the twin screw was $45 \mathrm{rpm}$. The composite containing $1.0 \mathrm{wt} \%$ MWCNTs was employed in this study. The pure PA66 was also extruded under the same conditions.

2.2. Surface Characterization and Property Tests. Fourier transformation infrared (FTIR) spectrometric measurements were performed by an FTIR spectrometer (TENSOR 27). The surface morphology of the cryofractured PA66/MWCNT composites was characterized by field emission scanning electron microscopy (FESEM).

The crystallization behaviors of the composites were carried out using a Q-100 (TA Instruments) differential scanning calorimeter (DSC) under nitrogen atmosphere to avoid any oxidation. The sample was heated from $40^{\circ} \mathrm{C}$ to $300^{\circ} \mathrm{C}$ at $10^{\circ} \mathrm{C} / \mathrm{min}$, held there for $5 \mathrm{~min}$ to erase any previous heating history, and then cooled from $300^{\circ} \mathrm{C}$ to room temperature (RT) at a cooling rate of $10^{\circ} \mathrm{C} / \mathrm{min}$.

The dry rolling-sliding friction tests were conducted using a twin disc wear testing machine. Full details of wear testing are similar to the previous work [14-16]. All discs were tested at the rotational speed of $1000 \mathrm{rpm}$, the applied load of $200 \mathrm{~N}$, and the slip ratio of $14.29 \%$ in a rolling-sliding contact.

Mechanical properties of the worn surfaces were determined using a nano-indentation/scratching tester (Micro Material Ltd). A Berkovich indenter with a tip diameter of $50 \mathrm{~nm}$ was employed throughout the experiment. For all indentation tests, the initial load was set to be $0.05 \mathrm{mN}$ and a constant maximum indentation load of $10 \mathrm{mN}$ was applied with the loading/unloading duration of 15 seconds. Distance between each indent was not less than $15 \mu \mathrm{m}$ in order to avoid any possible interference between neighboring indents. A test for each material was repeated for five times. Nano-hardness, reduced modulus, and elastic modulus were the average values of the results, and the presented loading-unloading curves were corresponding to the measured reduced modulus which was nearest to average value. The maximum relative standard deviation of the values for PA66, P-MWCNTs, AMWCNTs, and D-MWCNTs were 9.887\%, 2.145\%, 1.901\%, and $1.849 \%$, respectively.

\section{Results and Discussion}

3.1. Dispersibility of MWCNTs in PA66 Matrix. FESEM observation was used to qualitatively and directly visualize the state of dispersion of MWCNTs in the PA66 matrix. The representative FESEM images of the cross-sectional cryofractured surface of the PA66/MWCNT composites with $1.0 \mathrm{wt} \%$ differently modified MWCNTs are shown in Figure 1. The bright spots and lines in the FESEM images were attributed to MWCNTs as a result of their high electrical conductivity. The dispersion of P-MWCNTs was found to be poor in the PA66 matrix, as shown in Figure 1(a); furthermore, the exposed PMWCNTs were observed in the PA66 matrix. Therefore, it can be suggested that P-MWCNTs were mechanically mixed in the PA66 matrix so that the reinforcement effects of the MWCNTs have been weakened. It is unambiguous in 


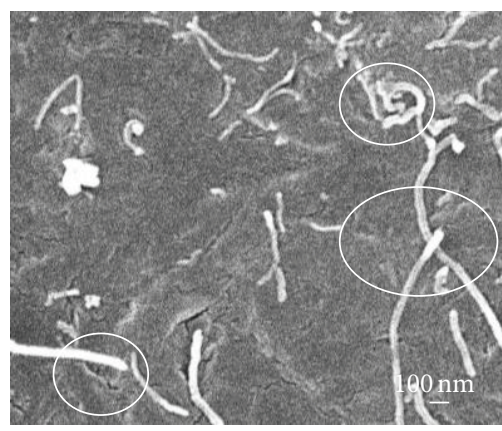

(a)

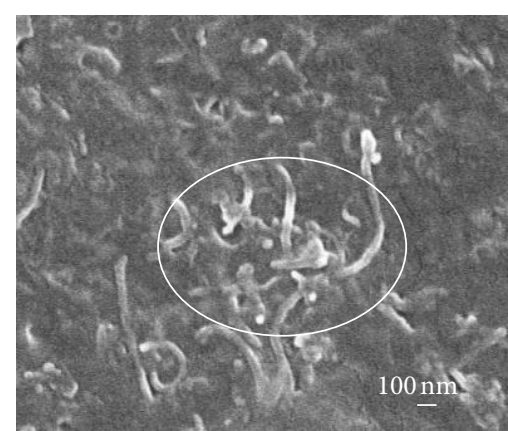

(b)

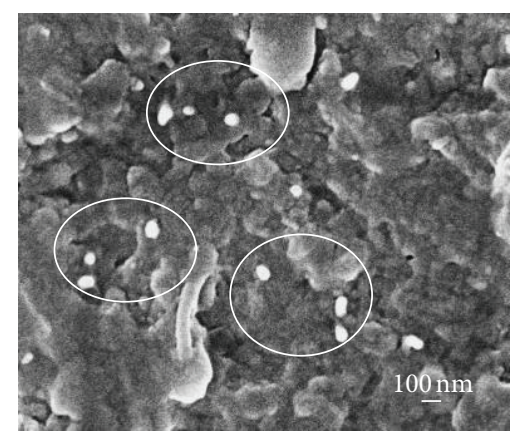

(c)

Figure 1: Fractured surfaces of PA66/MWCNT composites with 1.0 wt\% MWCNTs: (a) PA66/P-MWCNTs, (b) PA66/A-MWCNTs, and (c) PA66/D-MWCNTs.

Figure 1(b) that the A-MWCNTs were dispersed in the PA66 matrix without any exposed MWCNTs while some agglomerations of MWCNTs were observed in the PA66 matrix. The good dispersion could be attributed to the increased polarity of the A-MWCNTs by the carboxylic groups, resulting in the increased compatibility between MWCNTs and PA66 matrix. As shown in Figure 1(c), amine-modified MWCNTs were uniformly dispersed in PA66 matrix, and when cryofractured, some nanotubes were pulled out from PA66 matrix. The good interfacial compatibility should be attributed to the formation of the -NHCO- groups on the surface of MWCNTs which served as a link to improve the interface bonds between D-MWCNTs and PA66 matrix. With homogeneous dispersion of D-MWCNTs it is suggested that they were embedded, debundled, and distributed throughout the matrix as a result of effective functionalization. The dispersion state of MWCNTs in the PA66 matrix was the key parameter for drastic improvements of the crystal and mechanical properties.

To confirm the reaction between PA66 and D-MWCNTs during melt mixing, FTIR spectra of PA66, D-MWCNTs, and PA66/D-MWCNT composites were measured and are shown in Figure 2. The strong band of pure PA66 at $3296 \mathrm{~cm}^{-1}$ was attributed to hydrogen bonded $\mathrm{N}-\mathrm{H}$ stretching vibration. The absorption bands at $2933,2855 \mathrm{~cm}^{-1}$, and $1195 \mathrm{~cm}^{-1}$ resulted from the symmetric and asymmetric $\mathrm{C}-\mathrm{H}$ stretch vibrations and $\mathrm{C}-\mathrm{H}$ twisting. The amide $\mathrm{I}$ band at $1629 \mathrm{~cm}^{-1}$ was mainly associated with the $\mathrm{C}=\mathrm{O}$ stretching vibration. Amide II at $1533 \mathrm{~cm}^{-1}$ resulted from the $\mathrm{N}-\mathrm{H}$ bending and C$\mathrm{N}$ stretching vibration. The absorption band at $1273 \mathrm{~cm}^{-1}$ was due to $\mathrm{C}-\mathrm{N}-\mathrm{H}$ coupling vibration of the amide III band. In PA66/D-MWCNT composite, the band at $3440 \mathrm{~cm}^{-1}(\mathrm{O}-\mathrm{H}$ stretching vibration of alcoholic) and $1736 \mathrm{~cm}^{-1}(\mathrm{C}=\mathrm{O}$ symmetric stretching of carboxyl) in D-MWCNTs disappeared. It is suggested that D-MWCNTs were probably grafted on PA66 matrix by a condensation reaction during a meltcompounding process because this kind of reaction occurs easily between amine groups of D-MWCNTs' surface and carboxyl group of PA66. The same conclusion was also reached by other researchers $[17,18]$. Also, because of the similarity of the surface functional groups of D-MWCNTs and PA66 chains, good compatibility can be expected even though

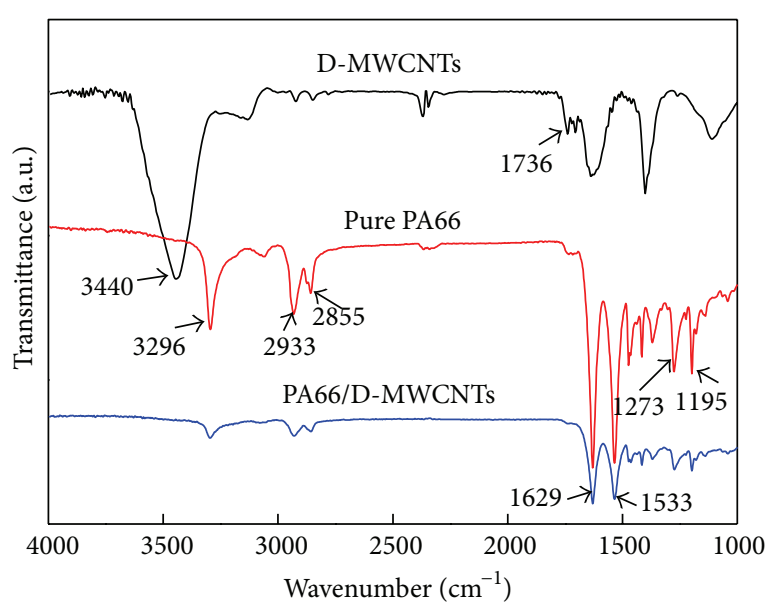

FIgURE 2: FTIR spectra of D-MWCNTs, pure PA66, and PA66/DMWCNT composites.

no condensation reaction occurs between D-MWCNTs and PA66. Therefore, we think the disappeared peaks are an indication of, at least in part, the condensation reaction.

From FESEM and FTIR results, it can be seen that DMWCNTs were uniformly dispersed in PA66 matrix and that molecular chain structure of PA66 showed no distinct difference owing to the chemical bonds between PA66 and D-MWCNTs.

3.2. Effects on PA66 Crystallinit. Usually, the crystallinity of polymer matrix significantly affects the friction property of the composites. Therefore, their crystallization behavior of PA66 matrix was investigated by DSC. Figure 3 shows the cooling thermograms of PA66 and PA66/MWCNT composites with $1.0 \mathrm{wt} \% \mathrm{MWCNTs}$ obtained at a $10^{\circ} \mathrm{C} / \mathrm{min}$ rate. It can be seen that the pure PA66 had only a crystallization peak temperature at $232^{\circ} \mathrm{C}$. The crystallization temperature shifted to a higher temperature, and the crystallization temperature range became broader with an introduction of MWCNTs, as had been reported in various polymer/CNTs composites [19, 20]. In general, inorganic fillers have two inconsistent influences on the crystallization of the semi-crystalline polymers. 
TABLE 1: Melting and crystallisation parameters of PA66 and PA66/MWCNT composites with 1.0 wt\% differently modified MWCNTs.

\begin{tabular}{lccccc}
\hline & $T_{m}\left({ }^{\circ} \mathrm{C}\right)$ & $\Delta H_{m}(\mathrm{~J} / \mathrm{g})$ & $X_{t}(\%)$ & $T_{c}\left({ }^{\circ} \mathrm{C}\right)$ & $\Delta H_{c}(\mathrm{~J} / \mathrm{g})$ \\
\hline Pure PA66 & 266.89 & 55.01 & 28.35 & 232.11 & 49.70 \\
PA66/P-MWCNTs & 263.48 & 64.64 & 33.31 & 244.11 & 45.04 \\
PA66/A-MWCNTs & 262.04 & 63.71 & 32.83 & 243.37 & 46.42 \\
PA66/D-MWCNTs & 266.07 & 66.91 & 34.48 & 240.76 & 34.19 \\
\hline
\end{tabular}

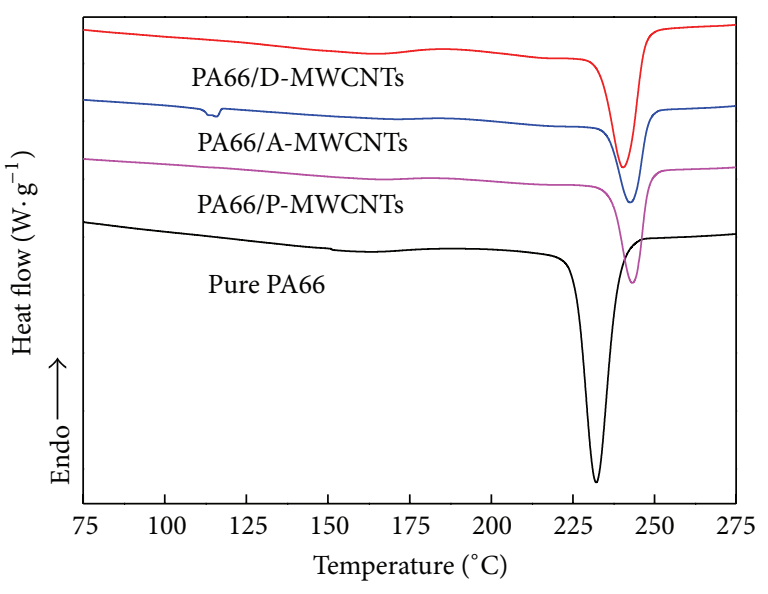

FIGURE 3: DSC thermograms on cooling of pure PA66 and PA66/ MWCNT composites with $1.0 \mathrm{wt} \%$ MWCNTs.

On one hand, they act as heterogeneous nucleating sites to facilitate the crystallization of polymers. On the other hand, they hinder the motion of polymer chain segments to retard the crystallization of polymers [18]. In the present work, the MWCNTs mainly acted as heterogeneous nucleating sites between the PA66 and MWCNTs and thus resulted in a higher crystallinity of the PA66 composites. However, the crystallization peak temperature of PA66/D-MWCNT composites was lower than that of the other composites. The low crystallization temperature of PA66/D-MWCNT composites could arise from polarity attraction or chemical interaction between amino end groups of D-MWCNTs and carboxylic groups of PA66.

Figure 4 shows the heating thermograms of PA66 and PA66/MWCNT composites with $1.0 \mathrm{wt} \%$ MWCNTs obtained at a $10^{\circ} \mathrm{C} / \mathrm{min}$ rate. With heating pure PA66, only one endothermic peak, which was associated with the lamellar thickness of PA66 [21], was observed at $266.7^{\circ} \mathrm{C}$. The introduction of MWCNTs into the PA66 matrix provided a large amount of nucleation sites for PA66 chains to restrained recrystallization or reorganization during the heating process in DSC testing [22], which resulted in only one melting peak of PA66. The changes in the characteristic parameters of the crystallization process are given in Table 1 . The crystallinity degree of pure PA66 was $28.35 \%$, while with $1.0 \mathrm{wt} \% \mathrm{D}$ MWCNTs incorporated in, the crystallinity degree increased to $34.48 \%$. As the crystallinity degree of the sample materials increased and their ductility reduced, these resulted in lowering the ability of the surface to accommodate the impacts and high strain rates in the dry sliding friction test. This is

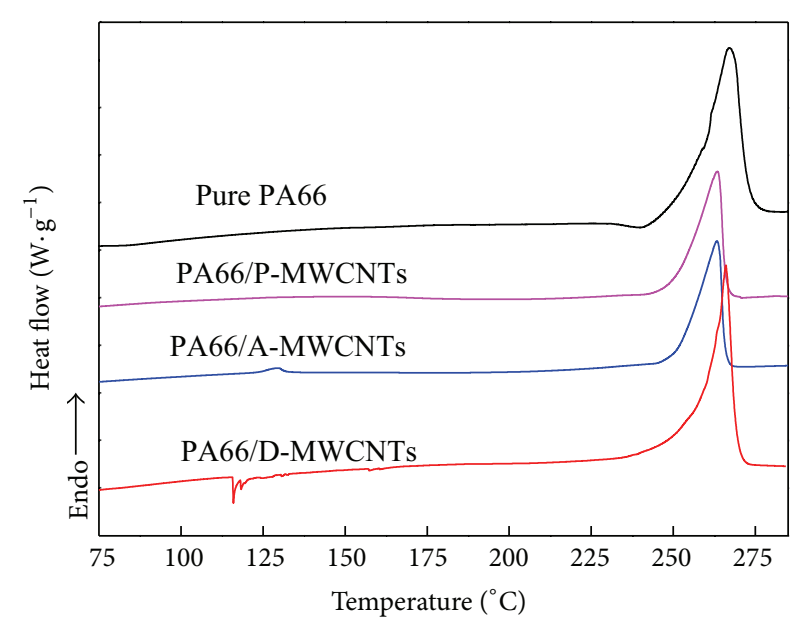

FIGURE 4: DSC thermograms on heating of pure PA66 and PA66/ MWCNT composites with $1.0 \mathrm{wt} \%$ MWCNTs.

similar to that an increase in crystallinity reduced the wear rate of the polymer-matrix composite [23]. However, it was also reported that a decrease of crystallinity reduced the wear rate of the composite [24,25]. This implies that the influence of the crystallization behavior, caused by an addition of the MWCNTs, is not the dominant factor for the enhancement of wear resistance.

3.3. Effects on Mechanical Properties. The mechanical properties of the composites, such as hardness, modulus, or the product of these factors, usually have significant influences on the wear behavior of the composites. According to previous research [26], materials with high hardness and high modulus would achieve lower friction coefficient. Thus, it is necessary to discuss the relationship between the mechanical properties and the tribo performance of polymer matrix composite.

A typical loading-unloading curve and the parameters used in an analysis of the nanoindentation test are shown in Figure 5. Among the mechanical properties which can be determined by a nanoindentation test, the elastic modulus $(E)$ and the nano-hardness $(H)$ are very common. They can be measured by analyzing the unloading part of the load-displacement curve. The nano-hardness $(H)$ can be computed as

$$
H=\frac{P_{\max }}{A},
$$

where $P_{\max }$ is the maximum normal load and $A$ is the contact area at the maximum load. 
TABLE 2: Mechanical properties deduced from the nanoindentation test.

\begin{tabular}{|c|c|c|c|c|c|c|}
\hline \multirow{2}{*}{ Specimen } & \multicolumn{2}{|c|}{ Reduced modulus (GPa) } & \multicolumn{2}{|c|}{ Elastic modulus (GPa) } & \multicolumn{2}{|c|}{ Hardness (GPa) } \\
\hline & Before test & After test & Before test & After test & Before test & After test \\
\hline PA66 & 2.9927 & 3.8911 & 1.7699 & 2.3029 & 0.2191 & 0.2779 \\
\hline PA66/P-MWCNTs & 3.0865 & 3.1707 & 1.8255 & 1.8755 & 0.2215 & 0.2335 \\
\hline PA66/A-MWCNTs & 3.2916 & 3.1028 & 1.9472 & 1.8352 & 0.2218 & 0.2293 \\
\hline PA66/D-MWCNTs & 3.0887 & 3.0938 & 1.8268 & 1.8299 & 0.2317 & 0.2271 \\
\hline
\end{tabular}

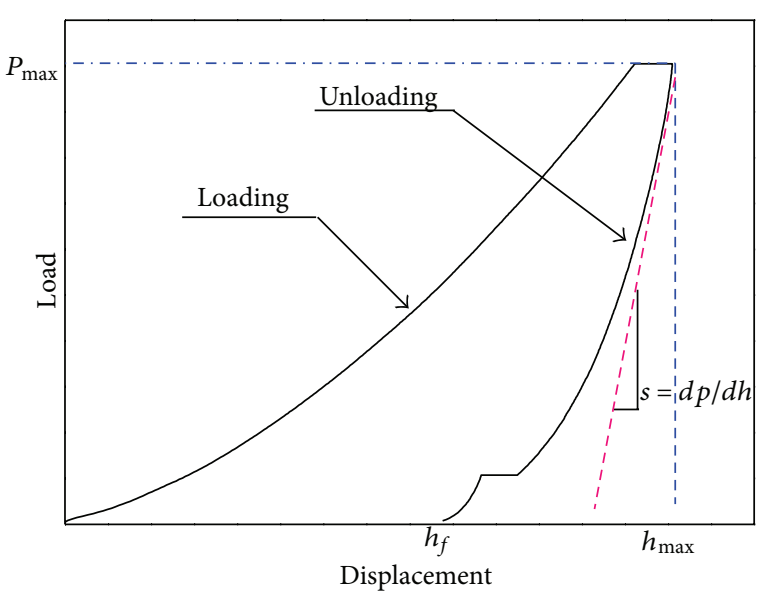

Figure 5: The loading and unloading curves in a nanoindentation test.

By considering the nonrigid indenters response, the elastic modulus $(E)$ can be directly calculated through the following equation:

$$
\frac{1}{E_{r}}=\frac{1-v^{2}}{E}+\frac{1-v_{i}^{2}}{E_{i}}
$$

where $E_{r}$ is the reduced modulus of indentation contact; Poisson's ratio $(v)$ of PA66 is 0.41 [27]. For the diamond indenter, the elastic modulus $\left(E_{i}\right)$ is $1140 \mathrm{GPa}$, and Poisson's ratio $\left(v_{i}\right)$ is 0.07 .

The reduced modulus of elasticity $\left(E_{r}\right)$ can be calculated from the unloading curve with Oliver-Pharr method [28] using the following equation:

$$
E_{r}=\frac{\sqrt{\pi}}{2} \frac{d p}{d h} \frac{1}{\sqrt{A}}
$$

where $d p / d h$ is the slope of the unloading curves.

Figure 6 gives loading-unloading curves, before and after a friction test, of pure PA66 and PA66/MWCNT composites with $1.0 \mathrm{wt} \%$ differently modified MWCNTs. These curves were obtained from the nanoindentation tests with a normal force of $10 \mathrm{mN}$. In Figure 6(a), before the friction test, the loading-unloading curves of specimens were shifted to the left, and maximum depth reduced with the addition of the modified MWCNTs, indicating that the composite's resistance to indentation gradually was increased with the addition of different modified MWCNTs. The indentation depths represent the contributions from the elasticity. This fact can be deduced from the unloading curves. With the introduction of differently modified MWCNTs the slope of unloading curve was increased. According to (3), there is a linear relationship between modulus and the slope of unloading (i.e., stiffness), and a growth in stiffness will result in a higher modulus.

As shown in Figure 7(a) pure PA66 was increased drastically after a frictional test. It is suggested that because of the low weathering property, PA66 became aging after the friction test, which led to the enhancement of the hardness and modulus. Figure 7(a) also shows a clear increasing trend of elastic modulus as the introduction of differently modified MWCNTs. Moreover, the hardness was also increased by adding modified MWCNTs into the polymer matrix, as illustrated in Figure 7(b). The effect of differently modified MWCNTs on the hardness and elastic modulus of composites from the nanoindentation tests is summarized in Table 2. The results of $H$ and $E$ in Table 2 are the averaged values in the depth. After friction testing, loading-unloading curves of specimen were shifted to the right, and maximum depth was increased with the addition of the modified MWCNTs, as shown in Figure 6(b). Figure 7 also shows that the composites with $1.0 \mathrm{wt} \% \mathrm{D}$-MWCNTs are not affected in the maximum depth, nano-hardness, and elastic modulus before and after the friction test. By contrast, the nano-hardness and elastic modulus of the pure PA66 are increased when D-MWCNTs have been added into polymer matrix because of the intrinsic high strength and low friction coefficient of D-MWCNTs and the strong interfacial adhesive force between D-MWCNTs and PA66 matrix. It is suggested that the addition of D-MWCNTs had a significant effect on enhancement of mechanical properties and is also expected to affect the tribological behavior of the composites.

3.4. Effect on Frictional Properties. In a similar manner, the tribological behavior of material has a close relation with its crystallization and mechanical properties. Figure 8 presents the friction coefficient of the PA66 and PA66 composites with differently modified MWCNTs during the tests at a speed of $1000 \mathrm{rpm}$, load of $200 \mathrm{~N}$, and slip ratio of $14.29 \%$. Figure 8 shows that the friction coefficient of PA66 was low at the early stage of the tribological test. This is because, at the initial stage, the contact surfaces between two discs were dominated by a bedding-in process where the contact between two surfaces occurred mainly in the microasperity level. Such a contact resulted in a lower friction force. When such micropeaks were worn out, friction coefficient increased gradually. As shown in Figure 8, the friction coefficient of the composites was the lowest when D-MWCNTs were added 


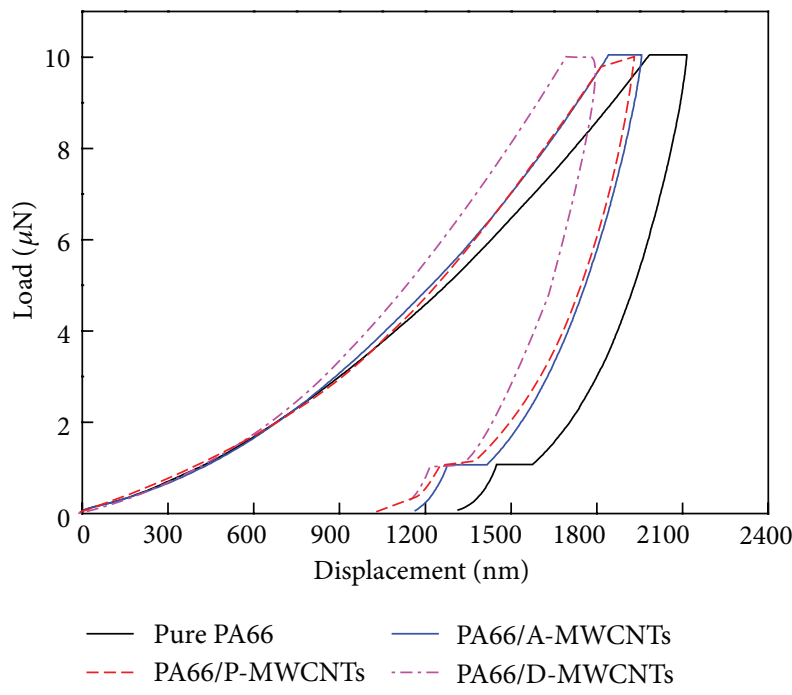

(a)

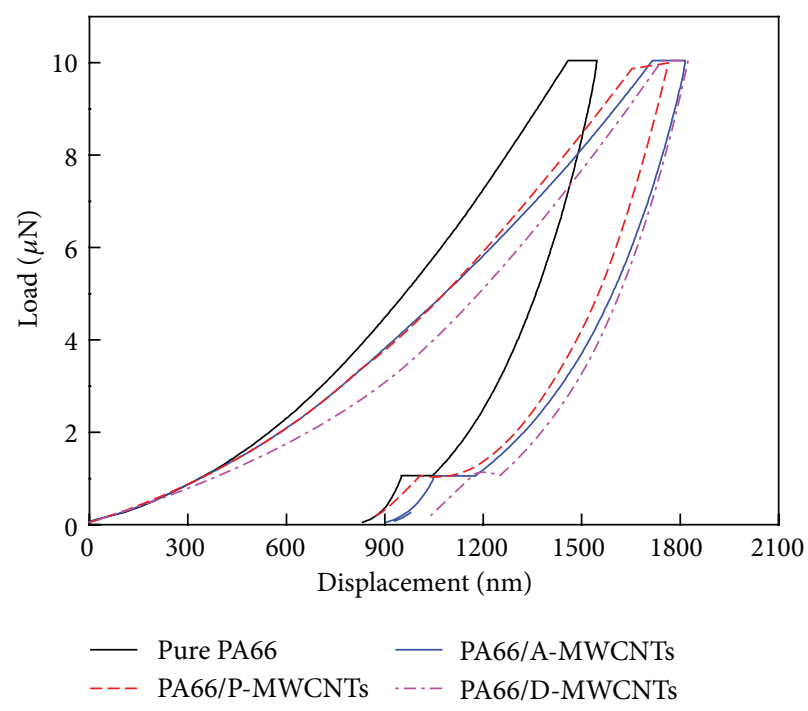

(b)

FIGURE 6: Loading-unloading curves of pure PA66 and PA66/MWCNT composites with 1.0 wt\% MWCNTs: (a) before friction test and (b) after friction test.

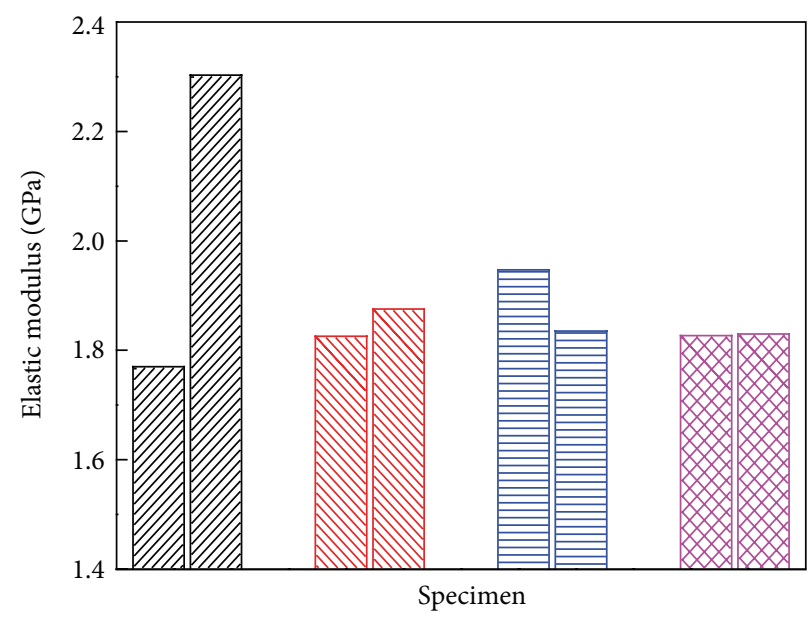

WIII Pure PA66 MIV PA66/P-MWCNTs PA66/D-MWCNTs

(a)
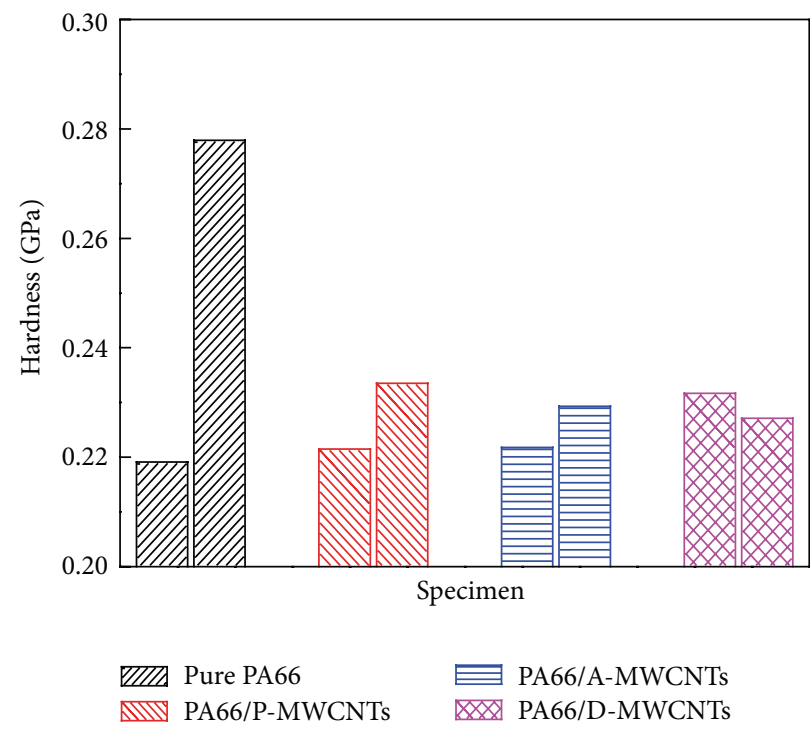

(b)

Figure 7: Elastic modulus (a) and nano-hardness (b) of PA66 and PA66/MWCNT composites with 1.0 wt\% MWCNTs (left: before friction test and right: after friction test).

to PA66 matrix. There is a significant reduction of $17 \%$ in frictional coefficient between pure PA66 and D-MWCNTs composites. This observation is very similar to that of Yoo and Norman [23]. High crystallinity matrices produced a transfer film on interfacial layer with low friction coefficients. During a sliding friction test in dry condition, the formation of the transfer film was always thought to be an important factor to enhance the wear resistance of the polymer composite. During the friction process, this layer film was continuously replenished as the composite worn, until the surface became saturated with MWCNTs fragments.
As shown in Figures 7 and 8, it is evident that, with an addition of D-MWCNTs, the nano-hardness of composite increases, but the friction coefficient of the composite decreases. It is suggested that the introduction of MWCNTs into polymer matrix improved abrasive resistance of composites owing to their load supporting role during a friction process. The load supporting role displayed on enhancement of the mechanical properties of the polymer. The same tendency of mechanical properties and friction behavior may be both ascribed to the different morphology and polymer/MWCNTs interactions with differently modified MWCNTs. 


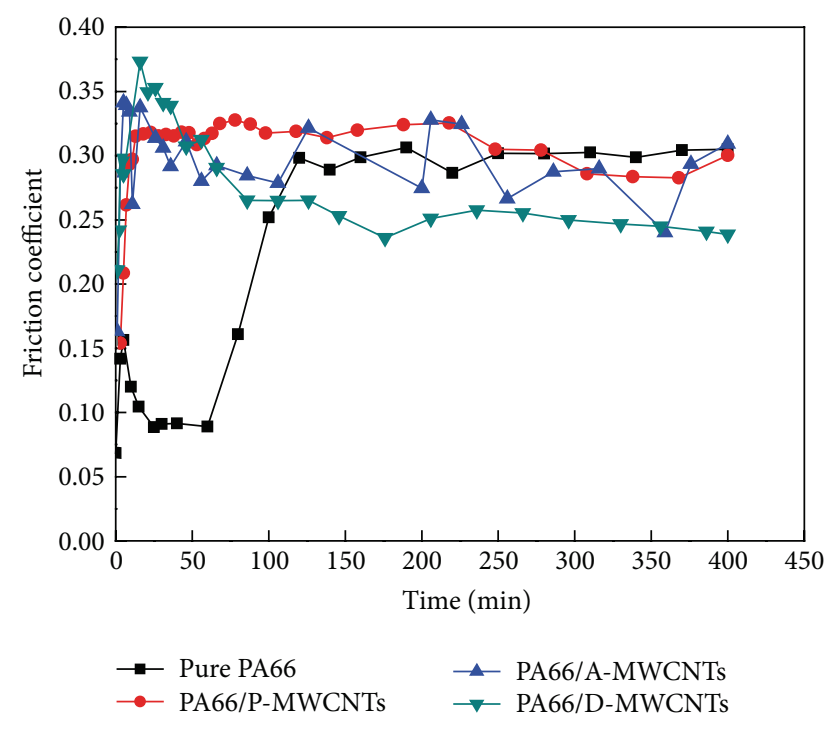

FIGURE 8: Friction coefficient curves for the pure PA66 and PA66/ MWCNT composites with $1.0 \mathrm{wt} \%$ differently modified MWCNTs (at $200 \mathrm{~N}, 1000 \mathrm{rpm}$, and $14.29 \%$ slip ratio).

\section{Conclusions}

In this work, the effects of modification methods on the dispersion of MWCNTs and the resulting mechanical and friction properties of the composites have been investigated and the following conclusions can be drawn.

Amine functionalization of MWCNTs improved the dispersion of MWCNTs in PA66 matrix. Because of the chemical bonds between PA66 and D-MWCNTs, molecular chain structure of PA66 showed no distinct difference. The degree of crystallization and melting enthalpy of PA66/MWCNTs were enhanced via the introduction of D-MWCNTs.

Before a friction test, surface chemical modification of MWCNTs resulted in an obvious increase in the nanohardness and elastic modulus of the composites. More interestingly, after the friction test, the nano-hardness and elastic modulus of the composites gradually reduced with the introduction of modified MWCNTs. The nano-hardness and elastic modulus of the PA66/D-MWCNT composites were almost unchanged, indicating the addition of D-MWCNTs effectively improved mechanical properties of the composites during the actual application process. The friction coefficient of the PA66/D-MWCNT composites was lowest of the all samples when D-MWCNTs were added to PA66 matrix.

\section{Conflict of Interests}

None of the coauthors have financial relationship with the commercial identities mentioned in this paper.

\section{Acknowledgments}

The authors acknowledge financial supports from Program for National Natural Science Foundation of China (21176169), Ph. D Programs Foundation of Ministry of Education of
China (20101402110007), and Research Project Supported by Shanxi Scholarship Council of China (2012-038).

\section{References}

[1] S. Iijima, "Helical microtubules of graphitic carbon," Nature, vol. 354, no. 6348, pp. 56-58, 1991.

[2] A. Thess, R. Lee, P. Nikolaev et al., "Crystalline ropes of metallic carbon nanotubes," Science, vol. 273, no. 5274, pp. 483-487, 1996.

[3] S. J. Tans, M. H. Devoret, H. Dai et al., "Individual single-wall carbon nanotubes as quantum wires," Nature, vol. 386, no. 6624, pp. 474-477, 1997.

[4] R. H. Baughman, A. A. Zakhidov, and W. A. De Heer, "Carbon nanotubes-the route toward applications," Science, vol. 297, no. 5582, pp. 787-792, 2002.

[5] T. X. Liu, I. Y. Phang, L. Shen, S. Y. Chow, and W. Zhang, "Morphology and mechanical properties of multiwalled carbon nanotubes reinforced nylon-6 composites," Macromolecules, vol. 37, no. 19, pp. 7214-7222, 2004.

[6] X. L. Xie, Y. W. Mai, and X. P. Zhou, "Dispersion and alignment of carbon nanotubes in polymer matrix: a review," Materials Science and Engineering R, vol. 49, no. 4, pp. 89-112, 2005.

[7] J. M. Augustine, S. N. Maiti, and A. K. Gupta, "Mechanical properties and crystallization behavior of toughened polyamide6/carbon nanotube composites," Journal of Applied Polymer Science, vol. 125, no. 1, pp. E478-E485, 2012.

[8] S. H. Jin, K. H. Yoon, Y. Park, and D. S. Bang, "Properties of surface-modified multiwalled carbon nanotube filled polyethylene terephthalate composite films," Journal of Applied Polymer Science, vol. 107, no. 2, pp. 1163-1168, 2008.

[9] F. C. Chiu and G. F. Kao, "Polyamide 46/multi-walled carbon nanotube nanocomposites with enhanced thermal, electrical, and mechanical properties," Composites A, vol. 43, no. 1, pp. 208-218, 2012.

[10] Z. Yang, S. Huang, and T. X. Liu, "Crystallization behavior of polyamide 11/multiwalled carbon nanotube composites," Journal of Applied Polymer Science, vol. 122, no. 1, pp. 551-560, 2011.

[11] H. Meng, G. X. Sui, G. Y. Xie, and R. Yang, "Friction and wear behavior of carbon nanotubes reinforced polyamide 6 composites under dry sliding and water lubricated condition," Composites Science and Technology, vol. 69, no. 5, pp. 606-611, 2009.

[12] I. Rodriguez-Pastor, H. Varela-Rizo, D. R. Bortz, G. Montes de Oca, I. Guinea, and I. Martin-Gullon, "Effects of processing and functionalization methods on nylon-6,6 nanocomposites with Helical-ribbon carbon nanofibers," Journal of Applied Polymer Science, vol. 126, no. 4, pp. 1437-1448, 2012.

[13] B. Krause, M. Ritschel, C. Täschner et al., "Comparison of nanotubes produced by fixed bed and aerosol-CVD methods and their electrical percolation behaviour in melt mixed polyamide 6.6 composites," Composites Science and Technology, vol. 70, no. 1, pp. 151-160, 2010.

[14] Y. K. Chen, S. N. Kukureka, and C. J. Hooke, "The wear and friction of short glass-fibre-reinforced polymer composites in unlubricated rolling-sliding contact," Journal of Materials Science, vol. 31, no. 21, pp. 5643-5649, 1996.

[15] S. N. Kukureka, C. J. Hooke, M. Rao, P. Liao, and Y. K. Chen, "Effect of fibre reinforcement on the friction and wear of polyamide 66 under dry rolling-sliding contact," Tribology International, vol. 32, no. 2, pp. 107-116, 1999. 
[16] Y. K. Chen, S. N. Kukureka, C. J. Hooke, and M. Rao, "Surface topography and wear mechanisms in polyamide 66 and its composites," Journal of Materials Science, vol. 35, no. 5, pp. 12691281, 2000.

[17] P. C. Ma, N. A. Siddiqui, G. Marom, and J. K. Kim, "Dispersion and functionalization of carbon nanotubes for polymer-based nanocomposites: a review," Composites $A$, vol. 41, no. 10, pp. 1345-1367, 2010.

[18] H. Meng, G. X. Sui, P. F. Fang, and R. Yang, "Effects of acid- and diamine-modified MWNTs on the mechanical properties and crystallization behavior of polyamide 6," Polymer, vol. 49, no. 2, pp. 610-620, 2008.

[19] J. Li, Z. P. Fang, L. F. Tong, A. Gu, and F. Liu, "Effect of multiwalled carbon nanotubes on non-isothermal crystallization kinetics of polyamide 6," European Polymer Journal, vol. 42, no. 12, pp. 3230-3235, 2006.

[20] Z. Y. Li, S. Z. Xu, W. T. Liu, S. Q. He, and C. S. Zhu, "Preparation and characterization of nylon610/functionalized multiwalled carbon nanotubes composites," Journal of Applied Polymer Science, vol. 113, no. 5, pp. 2805-2812, 2009.

[21] G. F. Shan, W. Yang, X. G. Tang et al., "Multiple melting behaviour of annealed crystalline polymers," Polymer Testing, vol. 29, no. 2, pp. 273-280, 2010.

[22] I. Y. Phang, J. Ma, L. Shen, T. Liu, and W. Zhang, "Crystallization and melting behavior of multi-walled carbon nanotubereinforced nylon-6 composites," Polymer International, vol. 55, no. 1, pp. 71-79, 2006.

[23] J. H. Yoo and S. E. Norman Jr., "Tribological behavior of blends of polyether ether ketone and polyether imide," Wear, vol. 162164, pp. 418-425, 1993.

[24] P. Werner, V. R. J. Altstädt, R. Jaskulka et al., "Tribological behaviour of carbon-nanofibre-reinforced poly(ether ether ketone)," Wear, vol. 257, no. 9-10, pp. 1006-1014, 2004.

[25] G. Y. Xie, Y. J. Zhong, G. X. Sui, and R. Yang, "Mechanical properties and sliding wear behavior of potassium titanate whiskersreinforced poly(ether ether ketone) composites under waterlubricated condition," Journal of Applied Polymer Science, vol. 117, no. 1, pp. 186-193, 2010.

[26] A. Molazemhosseini, H. Tourani, and M. R. Naimi-Jamal, "Nanoindentation and nanoscratching responses of PEEK based hybrid composites reinforced with short carbon fibers and nano-silica," Polymer Testing, vol. 32, no. 3, pp. 525-534, 2013.

[27] R. Jarrar, M. A. Mohsin, and Y. Haik, "Alteration of the mechanical and thermal properties of nylon 6/nylon 6,6 blends by nanoclay," Journal of Applied Polymer Science, vol. 124, no. 3, pp. 1880-1890, 2012.

[28] W. C. Oliver and G. M. Pharr, "Measurement of hardness and elastic modulus by instrumented indentation: advances in understanding and refinements to methodology," Journal of Materials Research, vol. 19, no. 1, pp. 3-20, 2004. 

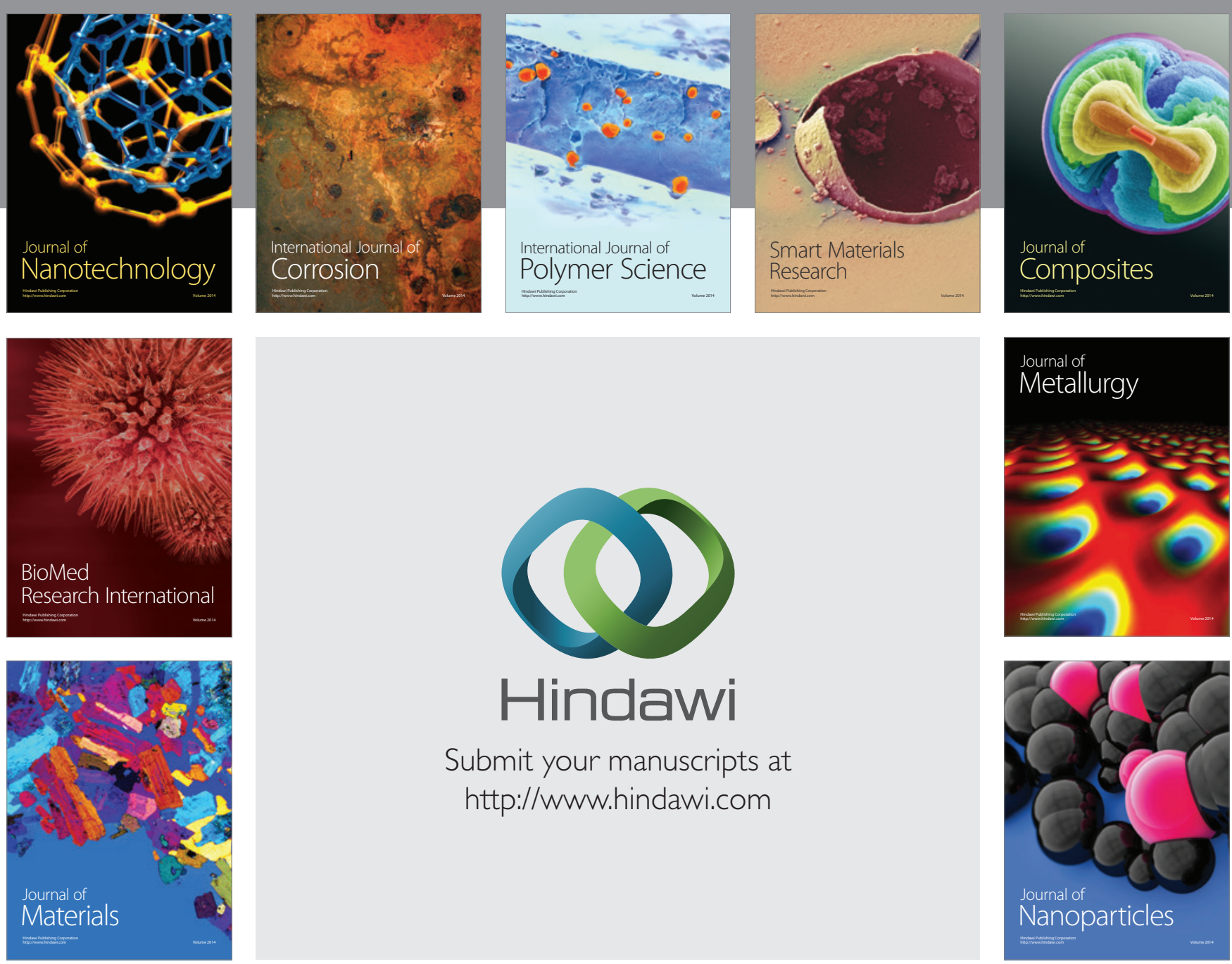

Submit your manuscripts at http://www.hindawi.com
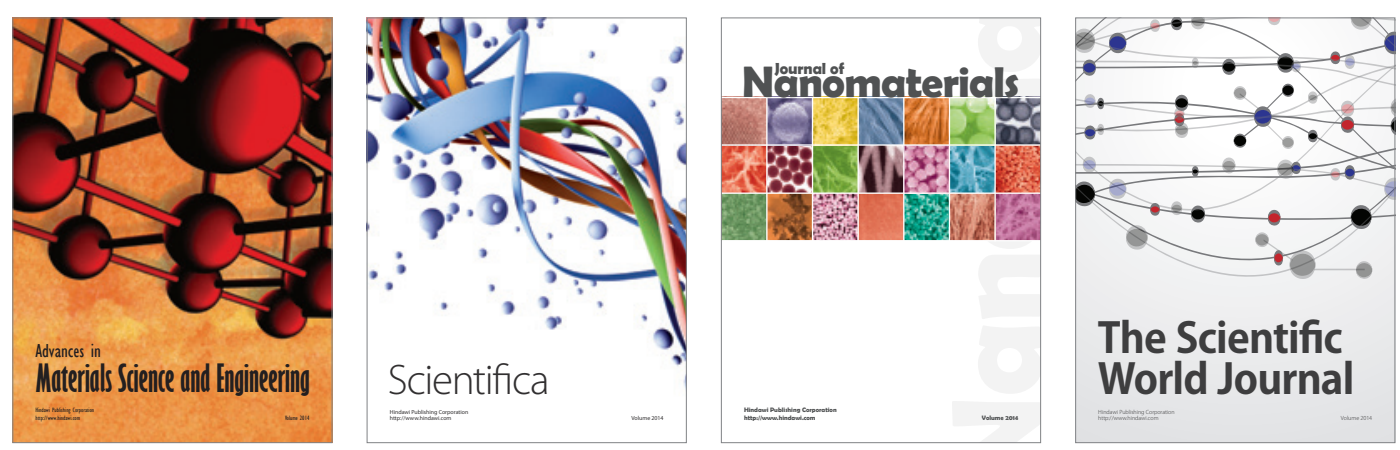

\section{The Scientific World Journal}
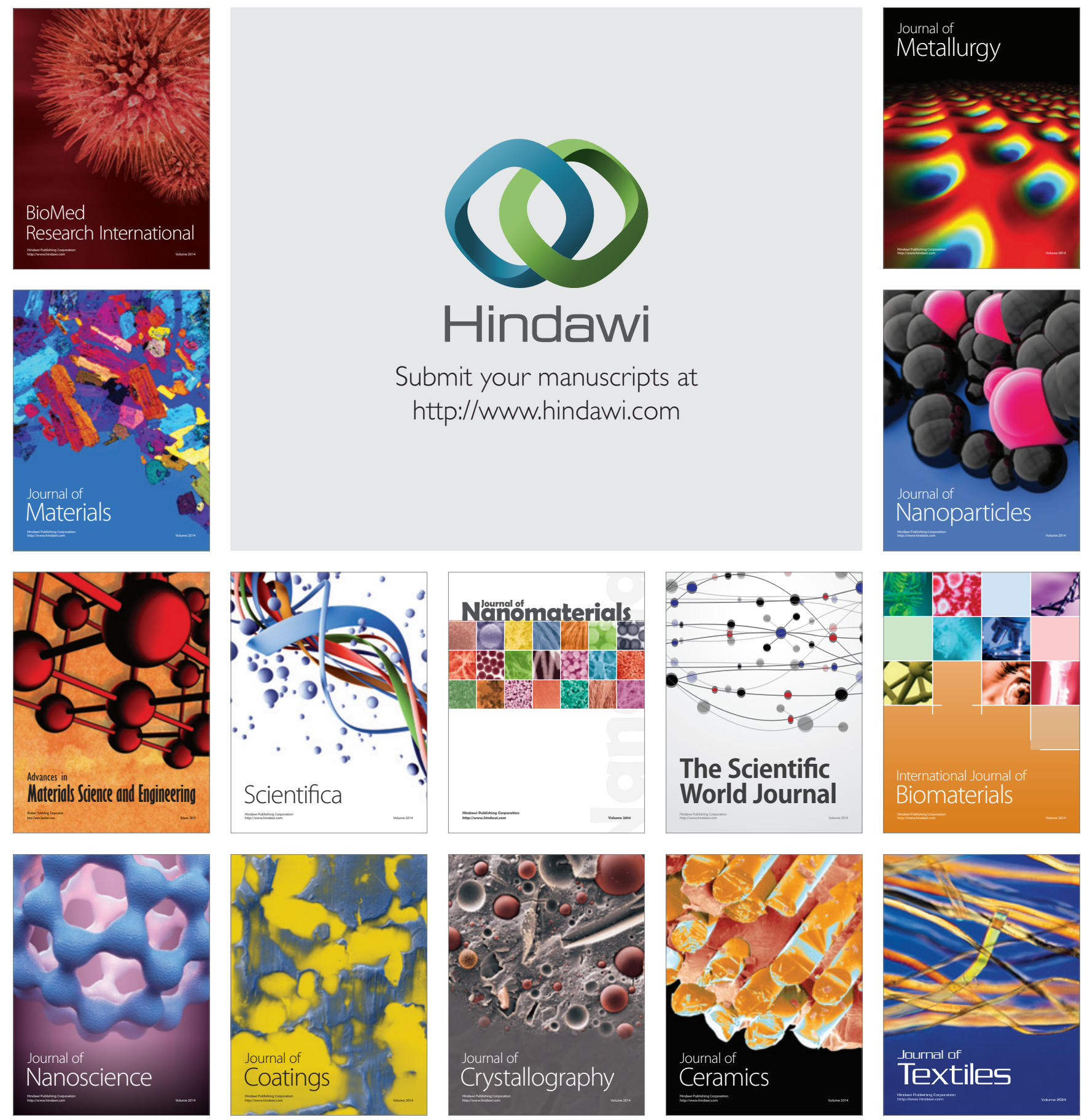